\title{
Neutrophil anti-neutrophil cytoplasmic autoantibody proteins: bactericidal increasing protein, lactoferrin, cathepsin, and elastase as serological markers of inflammatory bowel and other diseases
}

\author{
Kallirroi S. Kyriakidia , Vasileios E. Tsianos ${ }^{a}$, Evaggelos Karvounis ${ }^{a}$, Dimitrios K. Christodoulou, \\ Konstantinos H. Katsanos ${ }^{b}$, Epameinondas V. Tsianos ${ }^{c}$
}

University of Ioannina, Ioannina, Greece

\begin{abstract}
Inflammatory bowel disease (IBD) is a chronic inflammatory disorder of the gastrointestinal tract comprising Crohn's disease and ulcerative colitis. Although the pathogenesis of the disease is not clearly defined yet, environmental, genetic and other factors contribute to the onset of the disease. Apart from the clinical and histopathological findings, several serological biomarkers are also employed to detect IBD. One of the most thoroughly studied biomarker is anti-neutrophil cytoplasmic autoantibody (ANCA). We herein provide an overview of the current knowledge on the use of ANCA and certain ANCA proteins, such as bactericidal increasing protein, lactoferrin, cathepsin $\mathrm{G}$ and elastase, as serological markers for IBD and other diseases.
\end{abstract}

Keywords Inflammatory bowel disease, anti-neutrophil cytoplasmic autoantibody, bactericidal increasing protein, lactoferrin, cathepsin $\mathrm{G}$, elastase

Ann Gastroenterol 2016; 29 (3): 1-10

\section{Introduction}

The term inflammatory bowel disease (IBD) refers to a chronic, relapsing and tissue-destructive inflammatory disorder of the gastrointestinal tract an excessive recruitment of leukocytes from the blood circulation to the inflamed gut wall [1-3]. IBD mainly comprises ulcerative colitis (UC) and Crohn's disease (CD) [1]. Despite their common clinical features, UC and CD have significant clinical, endoscopic and histopathological differences [1]. Endoscopic findings,

aResearch Laboratory of Immunology (Kallirroi S. Kyriakidi, Vasileios E. Tsianos, Evaggelos Karvounis); ' Division of Gastroenterology (Dimitrios K. Christodoulou, Konstantinos H. Katsanos); ' (Epameinondas V. Tsianos), Faculty of Medicine, School of Health Sciences, University of Ioannina, Ioannina, Greece

Conflict of Interest: None

Correspondence to: Kallirroi Kyriakidi, Laboratory of Immunology, Faculty of Medicine, School of Health Sciences, University of Ioannina, Ioannina, Greece, Tel.: +30 26510 07806, +30 6947554145, e-mail: kalli491@yahoo.gr

Funding: This research project has been co-financed by the European Union (European Regional Development Fund, ERDF) and Greek national funds through the Operational Program "THESSALY, MAINLAND GREECE AND EPIRUS 2007-2013" of the National Strategic Reference Framework (NSRF 2007-2013)

Received 09 October 2015; accepted 26 February 2016; published online 24 March 2016

DOI: http://dx.doi.org/10.20524/aog.2016.0028 histological and laboratory investigations are used in order to define the extent and severity of inflammation, and the type of IBD [1]. During acute flares of IBD, neutrophil granulocytes and macrophages infiltrate the affected mucosa, manifested clinically by an increase in stool [4], and in macrophagederived inflammatory cytokines interleukin (IL)-1 $\beta,-6$, and tumor necrosis factor (TNF)- $\alpha$ [4].

Polymorphonuclear leukocytes (PMNs), also called neutrophils, are leukocytes with a crucial role for the innate immune response, as they protect the host from microbial pathogens and minimize deleterious effects mediated by dying or injured cells [5]. In IBD, infiltration of a large number of PMNs into the lamina propria represents a prominent histopathological feature [3]. Circulating PMNs are considered to be the first line of host defense against pathogens and their substances. Mature PMNs phagocytose, kill and digest microorganisms and their substances [3]. There are two major components of this defense mechanism: the degranulation where hydrolytic enzymes such as neutral proteases are released [2], and the initiation of the respiratory burst [3].

Conflicting studies show an impaired, normal, or enhanced burst of peripheral blood PMNs in patients with IBD [6]. It is possible that PMNs burst is more closely associated to the pathogenesis of UC than $\mathrm{CD}$, since the neutrophils are not involved in determining the classical lesions [6]. Actually, neutrophils' role is essential in UC inflammatory lesions and there is a high prevalence of anti-neutrophil cytoplasmic antibodies (ANCA) also in patients with UC in remission [6].

Several laboratory markers have been investigated for the diagnosis and differential diagnosis of IBD as well 
as for the assessment of disease activity and the risk of complications, the prediction of relapse and their effect on therapy [7]. Yet, the etiology of IBD has not yet been fully elucidated. Autoimmune processes may play a role in their pathogenesis, since several types of auto-antibodies and antibodies against external antigens have been found in IBD [2]. The serological immune response in IBD patients includes among others, autoantibodies, more commonly ANCA and their target antigens such as bactericidal increasing protein (BPI), lactoferrin, cathepsin G and elastase [1]. These proteins could be of high clinical utility, as they are non-invasive markers and could be valuable for the diagnosis, disease activity and future therapeutic line. Yet, these antigens are rarely considered specific for IBD, as they usually appear in other pathologic conditions, too.

\section{ANCA}

ANCAs are a group of autoantibodies, mainly of the immunoglobulin G (IgG) type, directed against cytoplasmic antigens localized in the azurophil and specific granules of neutrophils (the most common type of white blood cell) andmonocyte $[1,2,8-10]$. Therearetwo main types offluorescence pattern obtained on normal peripheral blood neutrophils: cytoplasmic granular with central interlobular accentuation (cANCA) and fine homogenous, diffuse rim-like staining of the perinuclear cytoplasm (or rim-accentuated fluorescence of the nuclei) designated as the pANCA pattern [11]. Besides the typical staining on indirect immunofluorescence (IIF), pANCA and CANCA, a third rim-like staining of the nuclear periphery often with multiple intranuclear foci was also identified and was designated as atypical pANCA $[1,2,11]$. The antigen specificity of atypical ANCAs is different from p/cANCA, as it is localized in the nuclear periphery, in contrast to the cytoplasmic location of p/cANCA [11].

ANCAs are detected as a blood test in a number of autoimmune disorders. The most commonly employed detection-method in use for ANCAs, is the screening method IIF on normal peripheral blood neutrophils [11]. However, for diagnostic purposes, enzyme-linked immunosorbent assay (ELISA) is also used in laboratories $[1,11]$. Since the target-antigens of ANCA are various different proteins and some of them are still unrecognized, thus sensitive and specific solid-phase methods cannot yet be developed [1].

ANCA are present in a number of chronic autoimmune disorders, such as IBD and vasculitides [1]. Serum levels of ANCA are used in the purposes of diagnosis, prognosis and in monitoring of inflammatory activity $[1,12]$. They are found, most notably, in rheumatoid arthritis (RA) and UC $[1,12]$. Atypical pANCA are commonly detected in patients with IBD, mainly in UC and to a lesser extent in CD, especially colonic CD [13], and some autoimmune liver diseases such as autoimmune hepatitis (AIH) and primary sclerosing cholangitis (PSC) [11].

Besides the standard methods for detection of ANCA, different diseases seem to yield better results, using one method specifically. For instance, in the systemic vasculitides, IIF on ethanol-fixed neutrophils is preferred. In IBD both IIF and fixed neutrophil ELISA is used. For the detection of ANCA in patients with PSC, immunoalkaline phosphatase staining method presented higher sensitivity [2]. Since it is difficult to differentiate atypical pANCA from typical vasculitis pANCA pattern on ethanol-fixed neutrophils, it is more reliable to use both ethanol and formalin-fixed neutrophil slides [11]. However, significant deviations can be identified among ANCA assays and commercially available substrates, which probably result from differences in cell preparation, fixation methodologies, and conjugates [11]. Moreover, IIF is a subjective technique and is highly dependent on the expertise of the observer. Consequently, different persons may observe the same pattern, but interpret the pattern differently. Despite the differences mentioned above, atypical pANCAs are associated to IBD and other autoimmune liver diseases, and are still often only referred to as pANCA [11]. This often leads to confusions regarding the type of ANCA and the exact target antigens of ANCA, detected in each pathologic condition [11]. That is the main reason why an alternative methodology, ELISA, was also developed for the detection of ANCA [11].

In 1990 two studies were held, one from Saxon et al [14] and the other one from Rump et al [15]. These two groups detected the presence of ANCA in the serum of patients with UC (50-90\%). Ever since, various studies have been conducted, proving the significance of ANCA in IBD, especially in UC, as the same percentages are quite lower in patients with CD (10-20\%) [2]. Another study detected ANCA in $45-82 \%$ of UC patients and $2-28 \%$ of CD patients [16]. As already mentioned, atypical pANCAs are not only detected in patients with UC, CD and PSC (63\%) [17], but also in a variety of other autoimmune liver diseases, such as $\mathrm{AIH}$, primary biliary cirrhosis (PBC) (6-39\%), viral hepatitis (11\%), and alcoholic hepatitis $(0-7 \%)[8,18]$. ANCAs are well established autoantibodies in systemic vasculitis, too [8]. In 2009, Papp et al presented ANCA patterns for IBD [19]. ANCAs were mainly appeared as atypical pANCA staining, both for UC (74.2-79.1\% of all ANCAs) and for CD (72.7$88.9 \%$ of all ANCAs) patients. In the immunofluorescence assay, these percentages were obviously lower [19]. pANCA patterns in UC and CD were $12.5 \%$ and $39.1 \%$ of all ANCAs, respectively [19]. While the prevalence of typical pANCA was high $(75.0 \%$ and $43.5 \%$ of all ANCAs, for UC and CD, respectively) [19].

For some research groups the term ANCA should be reconsidered. Billing et al proved with double-labeling IIF that the term ANCA is wrong for individuals with IBD and hepatobiliary disorders $[2,8]$. In fact, ANCA in those disorders presents a broad heterogeneous labeling of the nuclear periphery along with multiple intranuclear fluorescent foci. Two other research groups proved by immunoelectron microscopy, that this focal intranuclear fluorescence likely corresponds to invaginations of the nuclear envelope $[2,8]$. Therefore, for some the term ANCA, used in IBD and autoimmune liver disorders, is not that appropriate and should be replaced by the term antineutrophil nuclear antibodies $[2,10]$. 


\section{Utility of ANCA}

The role of ANCA alone, as diagnostic marker for IBD, appears to be limited because of its moderate sensitivity and its presence in other pathological conditions. The combination of pANCA and anti-Saccharomyces cerevisiae (S. cerevisiae) antibodies (ASCA) however, may be a useful diagnostic or prognostic tool for the distinction between CD and UC, when it is not obvious with the classic diagnostic tools (patient history, radiologic examination, endoscopy and biopsy) [11,20]. ASCA seems to be the most prominent member of anti-glycan antibodies. ASCA are anti-S. cerevisiae immunoglobulin (Ig) A and IgG antibodies [1]. They are believed to interact with mannose residues on mannan in the cell walls of $S$. cerevisiae. More specifically, the major antigen targeted with ASCA antibodies is a mannan, a cell wall glycoprotein, the 200 Kilo Dalton (KD) phosphopeptidomannan, of the common baker's or brewer's yeast $S$. cerevisiae $[1,12]$. Therefore, the ASCA+/ atypical pANCA- serologic pattern is mainly characteristic of $\mathrm{CD}$, while the ASCA-/atypical pANCA+ is characteristic of UC as well as Crohn's colitis [11,21]. However, the predictive value for the development of IBD, of ANCA alone is not clearly identified yet [22]. The results concerning the correlation between ANCA and response to therapy are conflicting. Some research groups state no correlation [11], while other groups claim that pANCA may be valuable for predicting response to anti-TNF, as negative status of pANCA is associated with early response to anti-TNF drugs [23]. The occurrence of atypical pANCA in UC is often associated with the clinical appearance, for instance some patients have a higher probability to develop a severe left-sided ulcerative colitis, more resistant to treatment, or a more aggressive course requiring surgery earlier. The presence of atypical pANCA in CD patients often appears as a UC-like colitis, with a left-sided inflammation of the colon. The atypical pANCAs in CD patients are associated to a later age of onset, while in patients with UC, no correlation was found with the activity of the disease [11].

\section{ANCA proteins}

ANCA recognize various targets in the neutrophils' constituent, such as proteins located in the granules of the neutrophils and monocytes, cytoplasmic proteins, proteins distributed in the cytoplasm and nuclei of eukaryotic cells and finally proteins located in the nuclei [1]. Yet, the triggering factor that disturbs the balance between immune tolerance and defensive inflammatory response to intestinal microbiota, still remains unknown [1].

ANCAs are classically associated with small-vessel systemic vasculitis, the so called ANCA-associated vasculitides $[1,8,9]$. In vasculitides, ANCA antibodies target different proteins, usually located in the lysosomes of monocytes and in the azurophilic granules of neutrophils $[1,10,12]$. Two of the first antigens identified in vasculitides were proteinase-3 (PR3) and myeloperoxidase (MPO), which are highly specific for
Wegener's granulomatosis and microscopic polyangiitis, Churg-Strauss syndrome, or idiopathic necrotizing crescentic glomerulonephritis, respectively $[1,2,8,9]$. PR3 along with neutrophil elastase and cathepsin G are three hematopoietic serine proteases stored in neutrophil cytoplasmic azurophilic granules $[10,24,25]$. PR3 mainly displays cANCA pattern and MPO is associated with the pANCA pattern $[1,2,10]$. PR3 and MPO do not seem to be specific for IBD, however, in a recent study PR3-ANCA were detected in 30/110 (27.3\%) of IBD patients [26]. PR3 reactivity was strikingly only detected in UC patients but not in CD [26]. In particular, of the $80 \mathrm{UC}$ patients tested, 30 (36.3\%) were PR3-ANCA positive, while of the $30 \mathrm{CD}$ patients tested, none was PR3-ANCA positive [26]. Additional studies need to be performed to validate this result.

Concerning IBD patients, four proteins located in the granules of the neutrophils and monocytes are of extended interest; these include two serine proteases cathepsin G and elastase, iron-binding protein lactoferrin and the natural antibiotic BPI. As mentioned above, neutrophil elastase, PR3, and cathepsin $G$ are three serine proteases. They help degrade engulfed microorganisms inside phagolysosomes. When the neutrophils are activated at inflammatory sites, these proteases are activated and play a key role in the regulation of inflammatory and immune responses, as well as in noninfectious inflammatory diseases $[1,24,25]$. It is likely that the target antigen for UC-related atypical pANCA is a complex conformational epitope, as yet unrecognized [1].

BPI, cathepsin G and elastase are sharply defined pANCA, while they can often be differentiated from true pANCA by their non-reactivity with formalin-fixed neutrophils. The cause of this is either the destruction of epitopes by formalin, or the leak from the cells because of their high solubility. These antigens are not only present in IBD, but also in other diseases, such as PSC and RA [10,30]. Moreover, pANCA elastase and lactoferrin may also occur in non-vasculitic conditions, e.g. RA and systemic lupus erythematosus (SLE), as mentioned below [26].

According to resent studies, a pANCA fluorescence, together with an intranuclear staining from patients with IBD and hepatobiliary disorders, proved that the target antigens are localized within the nucleus of neutrophilic granulocytes [1]. To further strengthen this hypothesis, sera from patients with UC, PCS, AIH or systemic vasculitis was examined on ethanol or formaldehyde-fixed neutrophils using confocal laser scanning microscopy and immunoelectron microscopy [8]. In immunoblotting experiments with nuclear envelope extracts of neutrophils, 92\% (59/64) of the serum samples positive for pANCA, also reacted with nuclear proteins (Table 1) [8].

\section{BPI}

BPI is a neutrophil granule protein acting as a natural antibiotic against Gram-negative bacteria [26,28]. Its strong anti-microbial action and anti-inflammatory features are based on its ability to detoxify lipopolysaccharides (LPS) of Gramnegative bacteria $[32,33]$, thereby preventing Gram-negative 
Table 1 Anti-neutrophil cytoplasmic autoantibody proteins in patients with ulcerative colitis

\begin{tabular}{|c|c|c|c|c|c|c|}
\hline Study & Method & UC patients & Lactoferrin (\%) & BPI (\%) & Cathepsin G (\%) & Elastase (\%) \\
\hline Nassberger et al [58] & ELISA & 54 & 3 & & & \\
\hline Peen et al [34] & ELISA & 24 & 50 & & & \\
\hline Mulder et al [33] & ELISA & 67 & 25 & & & \\
\hline Broekloelofs et al [59] & ELISA & 67 & 4 & & & \\
\hline Kossa et al [60] & ELISA & 49 & 41 & & 46 & 46 \\
\hline Yang et al [61] & ELISA & 36 & 8 & & & \\
\hline Stoffel et al [28] & ELISA & 54 & & 37 & & \\
\hline Sobajima et al [38] & ELISA & 35 & & & 26 & \\
\hline Walmsley et al [30] & ELISA & 52 & 4 & 29 & 4 & \\
\hline Sobajima et al [62] & ELISA & 60 & 13 & & 43 & \\
\hline Roozendaal et al [63] & ELISA & 96 & 26 & 2 & & \\
\hline Mayet et al [53] & ELISA & 60 & & & 38 & 3 \\
\hline Peen et al [34] & ELISA & 52 & 8 & & & \\
\hline Broekloelofs et al [59] & ELISA & 35 & 8 & & 0 & 0 \\
\hline Kossa et al [60] & ELISA & 33 & 3 & & 12 & 18 \\
\hline Yang et al [61] & ELISA & 37 & 3 & & & \\
\hline Stoffel et al [28] & ELISA & 44 & & 23 & & \\
\hline Roozendaal et al [63] & ELISA & 112 & 11 & 5 & & \\
\hline Locht et al [31] & ELISA & 30 & 13 & 20 & 23 & 0 \\
\hline Sugi et al [40] & ELISA & 33 & 69.7 & & 33.3 & \\
\hline Bahari et al [24] & ELISA & 108 & 31.5 & 5.6 & 7.4 & 25.9 \\
\hline
\end{tabular}

UC, ulcerative colitis; BPI, bactericidal increasing protein; ELISA, enzyme-linked immunosorbent assay

bacteria from activating neutrophils [31]. The functional domains of BPI, which bind to the inner core region of LPS, seem to trigger the neutralization of bacterial endotoxin and the delivery of endotoxin rich particles to host cells $[17,26]$. Another potential role of BPI is the stimulation of macrophages and the endothelium, thus procoagulant activity is expressed by the cells as well as proinflammatory cytokines [30]. Apart from BPI's protective role for the cell, it is also considered to be an ANCA antigen in various health disturbances [32,33]. However, it still remains difficult to determine the implications that lead to antibodies' secretion against neutrophil granule constituents, such as BPI [31].

BPI-ANCA staining could be either cytoplasmic or perinuclear fluorescence, probably according to the different target epitopes recognized in different diseases. While, perinuclear fluorescence appears when the smears are examined immediately and cytoplasmic when the smears are examined after $24 \mathrm{~h}$, presumably because BPI diffuses away from the nuclear membrane [27]. Usually, BPI produces a sharply defined pANCA without the nuclear extension. In patients with IBD, PSC and RA, BPI antibodies can often be differentiated from true pANCA by their non-reactivity with formalin-fixed neutrophils [27]. The reason for this staining is that formalin destroys the epitopes or because of the molecules' high solubility in the fixative, they leak from the cells [27].
The antigenicity of BPI could be destroyed by the presence of elastase and other serine proteases (e.g. PR3, cathepsin G), as it is susceptible to elastase cleavage. Consequently, if these proteins are subjected to protease cleavage, or denaturation, then the antigenicity of BPI is decreased [28].

A number of studies have been carried out to amplify the association of anti-BPI antibodies not only in IBD (mainly CD and less UC), but also in other diseases, such as PSC, vasculitides, idiopathic rheumatoid disorders etc. In 1997 Walmsley et al tested sera of IBD patients for IgG anti-BPI antibodies. Anti-BPI antibodies were present in $29 \%$ of patients with UC, $14 \%$ of patients with CD and $23 \%$ of patients with infectious enteritis. $60 \%$ of UC patients were ANCA positive and $28 \%$ of CD patients, respectively [30]. Anti-BPI antibodies were detected in $44 \%$ of ANCA-positive IBD patients [30]. Regarding UC patients, the presence of anti-BPI antibodies was related to disease activity, as well as a lower serum albumin concentration and a higher erythrocyte sedimentation rate. In CD patients, the relation referred to colonic involvement [30].

In a study conducted by Stoffel et al, serum samples from patients with IBD, vasculitides, PSC, RA and other diseases were obtained and ANCA and BPI antibodies were measured [28]. Immunofluorescence technique was performed for ANCA and ELISA to detect ANCA target antigens [28]. Of 54 patients with UC, BPI antibodies were detected in 20 (37\%), of whom 15 patients were ANCA-positive, and 5 ANCA-negative. 
Likewise, of 44 patients with CD, BPI antibodies were detected in 10 (23\%), of whom 5 patients were ANCA-positive, and 5 ANCAnegative. In patients with PSC, these results were respectively, 13/36 patients with BPI (36\%), 11 ANCA-positive and 2 ANCAnegative [28]. Thus, 20/59 patients with IBD were both positive for ANCA and BPI antibodies, while in 10/39 patients with IBD were detected BPI antibodies, though they were ANCA-negative [28]. In this study BPI and ANCA were found to be closely associated with IBD and PSC, but correlation was proven neither with ANCA-associated vasculitides nor with other idiopathic rheumatoid disorders. In particular, only $7 \%$ of all ANCAassociated vasculitides and only $6 \%$ of cANCA-positive sera (Wegener's granulomatosis, Churg-Strauss syndrome) tested were BPI-ANCA positive. On the contrary, $23 \%$ of pANCApositive samples from various chronic inflammatory disorders showed BPI-ANCA positivity [28].

Locht et al examined 30 serum samples from patients with UC, 30 with RA and 50 with reactive arthritis (ReA) (both acute and chronic disease) [26]. They used ELISA to detect anti-BPI antibodies and IIF for ANCA. Antibodies against BPI were found in $6 / 30(20 \%)$ patients with UC, 4/50 (8\%) patients with ReA, and $1 / 30$ (3\%) with RA [31]. There is a strong association between IBD and chronic ReA or ankylosing spondylitis (AS), as about $70 \%$ of patients with chronic ReA or AS had IBD [31].

More recently, in a study of Bahari et al, which consisted of 113 Iranian patients with UC, ELISA was used to find the target antigens for ANCA. Autoantibodies against lactoferrin, elastase, lysozyme, cathepsin G and BPI were detected. Of the 108 patients who completed the study auto-antibodies against BPI were detected in 5.6\% [24]. A pediatric study by Schultz et al on 27 cystic fibrosis (CF) patients, proved the existence of BPI-ANCA (IgG) in a high percentage $48 \%$. Three were also IgA BPI-ANCA-positive [29]. No clear answer could be given, as there was no considerable correlation to clinical data [29]. The role of BPI-ANCA to modulate opsonophagocytosis and disposal of Gram-negative bacteria and LPS, could enhance the possibility that they contribute to pulmonary pathology in pediatric CF patients [29]. These results were enhanced in 2008, when a high prevalence of anti-BPI in CF patients colonized with gram-negative bacteria was detected [17]. In PSC patients, anti-BPI has been found again in 5-46\% of the patients compared with $0-5 \%$ in healthy controls [17].

In 2010, Esquivel-Valerio et al tested 68 patients with pulmonary tuberculosis before and after treatment [32].
ANCAs by IIF were found in 3/68 (4.4\%) sera prior to treatment, all recognizing BPI. After treatment, this increased to $15 / 52(28.8 \%), 11 / 15(73 \%)$ directed against BPI [32]. They concluded that the prevalence of ANCA is modified by tuberculosis chemotherapy and that BPI is the main target antigen for ANCA in tuberculosis (Table 2) [32].

\section{Lactoferrin}

Lactoferrin is an iron-binding glycoprotein of the transferrin family. It occurs abundantly not only in specific granules of granulocytes, but also in tears, milk and secretions at mucosal surfaces, that makes lactoferrin a significant molecule in the first line of host defense [30,33-35]. Lactoferrin's properties are mainly related to the ability it exhibits, either to sequester iron in biological fluids or to destabilize the membranes of microbes [36]. Lactoferrin binds to the lipid A part of LPS [37]. It is also a prominent component of the secondary granules of PMNs and high levels of circulating lactoferrin imply active inflammatory disease [36,37]. Lactoferrin's resistance to proteolysis and degradation makes it a promising marker reflecting the intensity of the inflammatory process in the intestines [3].

The generation of hydroxyl radicals at sites of inflammation requires iron [37]. Lactoferrin exerts antibacterial effects by depriving bacteria from the iron required for their growth, through this iron binding mechanism lactoferrin can also prevent the formation of hydroxyl radicals, acting as a nonspecific anti-phlogistic defense factor [31,34-37]. Through its iron sequestering properties, lactoferrin can thus counteract the tissue-damaging effects of the oxygen free radicals [35,37]. The binding of lactoferrin by anti-lactoferrin antibodies increases hydroxyl radical formation by granulocytes [34]. Presence of such autoantibodies impairs the immune system and attenuates the antibodies' physiological actions [31].

Besides the anti-inflammatory properties, lactoferrin regulates the immune response and protects against infection and septic shock $[34,36]$. It may also be responsible for up- and downregulation of the immune cells and the cells involved in the inflammatory process [36]. Thus, it seems to have both pro- and anti-inflammatory activities [36].

Increased levels of plasma lactoferrin usually imply inflammation, infection, iron overload and tumor

Table 2 Bactericidal increasing protein-antibodies in patients with pathologic conditions

\begin{tabular}{|c|c|c|c|c|c|c|c|c|}
\hline \multirow[t]{2}{*}{ Study } & \multirow[t]{2}{*}{ Method } & \multicolumn{7}{|c|}{ Patients (\%) } \\
\hline & & UC & $\mathrm{CD}$ & $\mathrm{CF}$ & $\operatorname{ReA}$ & RA & PSC & $\mathrm{PBC}$ \\
\hline Stoffel et al [28] & ELISA & $20 / 54(37)$ & $10 / 44(23)$ & & & & $13 / 36(36)$ & \\
\hline Walmsley et al [30] & ELISA & $15 / 52(29)$ & $5 / 36(14)$ & & & & & \\
\hline Locht et al [31] & ELISA & $6 / 30(20)$ & & & $4 / 50(8)$ & $1 / 30(3)$ & & \\
\hline Schultz et al [29] & ELISA & & & $16 / 27(59)$ & & & & \\
\hline Lindgren et al [64] & ELISA & & & & & & $20 / 76(26)$ & $20 / 100(20)$ \\
\hline
\end{tabular}

UC, ulcerative colitis; CD, Crohn's disease; CF, cystic fibrosis; ReA, reactive arthritis; RA, rheumatoid arthritis; PSC, primary sclerosing cholangitis; PBC, primary biliary cirrhosis; ELISA, enzyme-linked immunosorbent assay 
development [35]. During inflammation, lactoferrin levels in biological fluids may greatly increase indicating a marker for inflammatory diseases. A normal plasma concentration can be as low as $0.4-2 \mathrm{mg} / \mathrm{L}$, but increases up to $200 \mathrm{mg} / \mathrm{L}$ in septicemia [36].

In ReA, the inadequate handling of foreign bacterial antigens boosts pathogenesis [31]. According to some investigators, anti-lactoferrin antigens seems to be a major target in UC [31]. Peen et al analyzed 52 serum samples from patients with CD, 24 from patients with UC, and 12 from patients with PSC. They analyzed serum samples for the presence of anti-lactoferrin antibodies by ELISA [34]. Antibodies against lactoferrin produce a pANCA pattern owing to perinuclear/nuclear localization of lactoferrin after ethanol fixation [34]. IgG antilactoferrin antibodies had 12 of 24 (50\%) patients with UC, $4 / 52$ CD patients ( $8 \%$ ), all four of whom had disease involving colon, and finally $6 / 12$ patients with PSC (50\%), all of whom had also UC [34]. The occurrence of IgG anti-lactoferrin was also confirmed by Western blotting [34]. IgA anti-lactoferrin was detected in $10 \%$ of patients' sera (5 of 51), specifically, in 7 of 21 (33\%) UC patients and in only 1 of 11 (9\%) PSC patients [34].

In another study [33], polyclonal anti-lactoferrin antibodies in the sera of patients with $\mathrm{UC}$ and $\mathrm{CD}$, recognizing the $77-\mathrm{kD}$ fragment were directed against lactoferrin [33]. Serum samples were tested with IIF and Western blotting. Of 67 UC patients, 15 were positive for lactoferrin and 11 of them were also pANCA-positive [33]. Six of $35 \mathrm{CD}$ patients were positive for lactoferrin and 2/6 were also pANCA-positive [33]. All the samples positive for lactoferrin by ELISA had been scored as positive for anti-lactoferrin by Western blotting, too [33].

In a study held by Sobajima et al [38], 35 patients with UC were analyzed for their specificity of ANCA and 25 of them were positive. Moreover, sera of these patients was tested with ELISA for anti-lactoferrin antibodies. Anti-lactoferrin antibodies were detected in $9 \%(3 / 35)$ of the patients and they were also positive for pANCA $(12 \%, 3 / 25)$. However, in Western blotting using lactoferrin, none of the serum samples which were positive for ELISA showed any positive bands. The antigenic determinants of lactoferrin appear to be sensitive to such denaturation procedures as those employing 2-mercaptoethanol and Sodium dodecyl sulfate. This also appears to be the case with lactoferrin [38].

Roozendaal et al [39] studied the presence of anti-lactoferrin antibodies in IBD and PSC patients. Lactoferrin was not related to treatment, disease activity, duration of disease, or disease extent. Antibodies against human lactoferrin were detected by ELISA and by immunoblotting, using an extract of sonicated neutrophils as antigen source. Autoantibodies to lactoferrin were found in $29 \%$ of patients with UC, $13 \%$ of patients with $\mathrm{CD}$, and $22 \%$ of patients with PSC [39]. They concluded that the presence of autoantibodies to lactoferrin for patients with PSC did not exhibit any correlation with the duration of the disease or the presence of cirrhosis. However, autoantibodies to lactoferrin seem to be a common feature of IBD and PSC, with limited sensitivity and specificity [39].

Two studies mentioned before, concerning also BPI, referring to IBD patients and antibodies against lactoferrin, showed high percentages of these antibodies [24,40]. 23 of $33(69.7 \%)$ UC patients and 21 of 31 (67.7\%) CD patients had anti-lactoferrin antibodies [40]. In a more resent study of Bahari et al, of 108 Iranian patients with UC, auto-antibodies against lactoferrin were detected in $31.5 \%$ [24].

Locht et al studied the presence of anti-lactoferrin antibodies in the sera of patients with UC, ReA, RA and AS [31,37]. Positive for anti-lactoferrin antibodies were: 4/30 (13\%) patients with UC, 10/50 (20\%) patients with ReA and $2 / 30(7 \%)$ with RA [31]. Of 55 ReA patients, 31 were positive for IgG-ANCA and of them 23 (56\%) had antilactoferrin antibodies, measured by ELISA [37]. Similar studies have been carried out, reporting the prevalence of anti-lactoferrin in several autoimmune diseases including RA, SLE, ReA and AS [17]. Moreover, antibodies against lactoferrin were present in $20 \%$ of patients with $\mathrm{AIH}, 23 \%$ of patients with PBC, $22 \%$ of patients with PSC, $13 \%$ of patients with alcoholic cirrhosis, $3 \%$ of patient with chronic hepatitis $\mathrm{B}$, and $3 \%$ of patient with chronic hepatitis C. Antibodies against lactoferrin were associated with lower albumin levels in PSC [41].

Teegen et al presented lactoferrin as the main target antigen for UC-associated pANCA on the basis of its interaction with DNA [42]. They concluded that autoantibodies against DNA-bound lactoferrin probably correlate with composites of chromatin and granulocyte proteins, the so-called neutrophil extracellular traps, which represent a new building block of the innate immunity $[25,42]$. Various studies have been held, proving the use of fecal lactoferrin as a useful marker in IBD (Table 3) [43-48].

\section{Cathepsin G}

Dewald et al, Rindler Ludwig and Braunsteiner, in 1975, found another protease retaining enzymatic activity on synthetic substrates for chymotrypsin, cathepsin G [25]. Cathepsin G along with neutrophil elastase and PR3, are three serine proteases, which constitute neutrophil azurophilic granules and participate in the nonoxidative pathway of intracellular and extracellular pathogen destruction $[25,48,49]$. Serine proteases are present in eukaryotes, prokaryotes, bacteria, and archaea [25]. During anti-inflammatory response, these molecules retain the delicate balance between tissue protection and destruction [49]. Their role in the neutrophils is crucial, as they are involved in the destruction of internalized pathogens, the modification of chemokines and cytokines, the activation of cell surface receptors and finally apoptosis $[49,50]$. They include different types of molecules, such as digestive enzymes, clotting factors, leukocyte granule-associated proteases, with a diverse biological function for each one. Cathepsin $\mathrm{G}$ is a serine protease that makes up approximately $20 \%$ of the azurophilic granule proteins of the neutrophils. Its roles and biological actions are many, as it contributes in neutrophil function during inflammatory processes, including degradation of extracellular matrix components and cytokines, modulation of integrin clustering on neutrophils, and direct chemoattraction of T cells and other leukocytes [48]. 
Table 3 Lactoferrin antibodies in patients with pathologic conditions

\begin{tabular}{|c|c|c|c|c|c|c|c|c|c|}
\hline \multirow[t]{2}{*}{ Study } & \multirow[t]{2}{*}{ Method } & \multicolumn{8}{|c|}{ Patients (\%) } \\
\hline & & $\mathrm{CD}$ & UC & PSC & $\operatorname{ReA}$ & RA & $\mathrm{CF}$ & $\mathrm{AIH}$ & PBC \\
\hline Locht et al [37] & ELISA & & & & $23 / 55(42)$ & $3 / 40(7)$ & & & \\
\hline Peen et al [34] & ELISA & $12 / 24(50)$ & $4 / 52(8)$ & $6 / 12(50)$ & & & & & \\
\hline Locht et al [31] & ELISA & $4 / 30(13)$ & & & $10 / 50(20)$ & $2 / 30(7)$ & & & \\
\hline Schultz et al [29] & ELISA & & & & & & $1 / 27(3.7)$ & & \\
\hline Roozendaal et al [41] & ELISA & & & $12 / 55(22)$ & & & & $18 / 88(20)$ & $12 / 53(23)$ \\
\hline Lindgren et al [64] & ELISA & & & $17 / 76(22)$ & & & & & $7 / 100(7)$ \\
\hline Teegen et al [42] & ELISA & & $2 / 39(5.1)$ & & & & & & \\
\hline
\end{tabular}

CD, Crohn's disease; UC, ulcerative colitis; PSC, primary sclerosing cholangitis; ReA, reactive arthritis; RA, rheumatoid arthritis; CF, cystic fibrosis; AIH, autoimmune hepatitis; PBC, primary biliary cirrhosis; ELISA, enzyme-linked immunosorbent assay

According to some studies, the cathepsin G cleavage depends on protein-coupled protease-activated receptors, the so-called PARs. These receptors can be cleaved by different proteases from the circulation, inflammatory cells, digestive glands and microorganisms $[48,49]$. During injury and disease, such signaling proteases are generated and are involved in inflammation, hemostasis, neurotransmission, cell migration, and division [48].

Although cathepsin $\mathrm{G}$ is one of the most abundant proteins in human neutrophils, its role in pathogenesis still remains unknown. Evidence from different research groups involves cathepsin G in the pathophysiology of IBD and marks it as a new target for IBD therapy [51]. Dabek et al proved an overexpression of cathepsin $G$, using real-time polymerase chain reaction and Western blotting techniques and thus concluded that cathepsin $\mathrm{G}$ may be an important factor in pathogenesis of IBD [48]. Even though there are no clear evidences for the pathogenesis of cathepsin $G$ as an antigen processing enzyme, the last one seems to be associated with a number of inflammatory processes, such as RA [52], various lung disorders, for instance CF [25], acute lung injury [25], acute respiratory distress syndrome etc. [25].

Mayet et al examined 15 patients with active UC and 60 patients with $\mathrm{CD}$, using ELISA and Western blotting, to detect cathepsin $G$ antibodies in patients' sera [53]. In the sera of 60 patients with $\mathrm{CD}$, antibodies against cathepsin $\mathrm{G}$ were detected in 24 serum samples [53]. Though, Mayet et al found no correlation between cathepsin G and UC, no patient with UC was positive for cathepsin G. Four years later in 1996, Sobajima et al [38], in a study mentioned above, detected antibodies against cathepsin G in 26\% (9/35) of patients with UC [38]. Of them, 7 patients were also pANCA-positive, one was nANCA (nuclear staining pattern)-positive and 1 was ANCA-negative [38]. Twelve of 25 ANCA-positive sera reacted with cathepsin $G$, yet the reactive antigens were unclear in the remaining sera. In Western blotting, cathepsin G seems to be significantly correlated with another antigen, a $28-\mathrm{kD}$ protein. But this study did not demonstrate any significant results [38]. Almost a year later, Sobajima et al revealed two proteins, one $28 \mathrm{kD}$ and the other $29 \mathrm{kD}$, which corresponded to highmobility group (HMG) protein 2 and HMG1, respectively [54].
In the aforementioned research by Locht et al [31], antibodies against cathepsin were detected in $7 / 30(23 \%)$ patients with UC, $1 / 50$ (2\%) patients with ReA, and 4/30 (13\%) patients with RA [31]. Kuwana et al highlighted the presence of pANCA, associated with antibodies against cathepsin G, in the sera of patients with UC. The results of IIF in 32 patients with active UC, showed a high percentage $62.5 \%$ of pANCA. Antibodies to cathepsin $\mathrm{G}$ were detected by ELISA and Western blotting in $40.6 \%$ of 32 patients with active UC. According to endoscopy, their prevalence was significantly higher in patients with severe colitis than in those with mild or moderate colitis. Kuwana et al concluded that the prevalence and titers of anti-cathepsin $\mathrm{G}$ antibodies were significantly higher during the active than the inactive phase of the disease, thus titers of anti-cathepsin $G$ could be useful for evaluating the activity of UC [55].

Various other studies have been carried out through the years. A more resent one is this of Bahari et al, mentioned before, in which, of 108 Iranian patients with UC, autoantibodies against cathepsin were detected in only $7.4 \%$ of the population [24]. Also, antibodies against cathepsin G were found in up to $35 \%$ of patients with PSC, of whom $80 \%$ were diagnosed with IBD (Table 4) [17].

\section{Elastase}

Elastase, PR3, and cathepsin G are three homologous proteases that belong to the chymotrypsin superfamily of serine proteases [25]. These proteases constitute neutrophil azurophilic granules and participate in the nonoxidative pathway of intracellular and extracellular pathogen destruction $[25,48,49]$. Elastase or otherwise human neutrophil elastase was identified in 1968 by Janoff and Scherer [25]. Its name "elastase" was given in association with elastin, as in the beginning, elastase was known as an enzyme capable of releasing soluble peptides from insoluble elastin [25]. Nowadays, we are aware that its activities are not always physiologically related to the digestion of this target [25].

The roles and the mechanisms of elastase are quite similar to those of cathepsin G [25]. Human PMNs release 
Table 4 Cathepsin $\mathrm{G}$ antibodies in patients with pathologic conditions

\begin{tabular}{|c|c|c|c|c|c|c|c|}
\hline \multirow[t]{2}{*}{ Study } & \multirow[t]{2}{*}{ Method } & \multicolumn{6}{|c|}{ Patients (\%) } \\
\hline & & UC & $\mathrm{CD}$ & $\operatorname{ReA}$ & RA & PSC & $\mathrm{PBC}$ \\
\hline Mayet et al [53] & ELISA & $0 / 15(0)$ & $24 / 60(40)$ & & & & \\
\hline Sobajima et al [38] & ELISA & $9 / 35(26)$ & & & & & \\
\hline Locht et al [31] & ELISA & $7 / 30(23)$ & & $1 / 50(2)$ & $4 / 30(13)$ & & \\
\hline Kuwana et al [55] & ELISA & $13 / 32(40.6)$ & & & & & \\
\hline Lindgren et al [64] & ELISA & & & & & $20 / 76(37)$ & $4 / 100(4)$ \\
\hline Bahari et al [24] & ELISA & $8 / 108(7.4)$ & & & & & \\
\hline
\end{tabular}

UC, ulcerative colitis; $C D$, Crohn's disease; ReA, reactive arthritis; RA, rheumatoid arthritis; $P S C$, primary sclerosing cholangitis; PBC, primary biliary cirrhosis; ELISA, enzyme-linked immunosorbent assay

Table 5 Elastase antibodies in patients with pathologic conditions

\begin{tabular}{|c|c|c|c|c|c|c|}
\hline \multirow[t]{2}{*}{ Study } & \multirow[t]{2}{*}{ Method } & \multicolumn{5}{|c|}{ Patients } \\
\hline & & UC & $\operatorname{ReA}$ & RA & PSC & PBC \\
\hline Locht et al [31] & ELISA & $0 / 30(0)$ & $1 / 50(2)$ & $0 / 30(0)$ & & \\
\hline Lindgren et al [64] & ELISA & & & & $14 / 76(18.4)$ & $15 / 100(15)$ \\
\hline Bahari et al [30] & ELISA & 28/108 (25.9) & & & & \\
\hline
\end{tabular}

elastase and other granule constituents in response to a variety of stimuli [56]. Among the degradative proteases, elastase is the most prominent constituent of the azurophil granules of PMNs. Elastase degrades structural and soluble proteins at physiological $\mathrm{pH}$ and could serve as a marker for degranulating activity of PMNs outside the cells [6]. Elastase exhibits antimicrobial activity as a serine esterase and plays an important role in the pathogenesis of inflammatory disorders [5,57]. Not surprisingly, elastase's activity is elevated in both colonic mucosa and blood in IBD patients [57]. The same elevated levels appear in stool samples of patients with IBD, leading to the assumption that elastase can act as an aggravating factor in IBD [5,57]. Elastase contributes to tissue destruction generally in inflammatory diseases, as a product secreted by activated neutrophils. Hence, besides IBD, it is also implicated to acute respiratory distress syndrome, lung emphysema, and RA [57].

Shioya et al provided evidence for the usefulness of elastase-inhibitors in clinical colonic inflammation [57]. They concluded that its activity in IBD patients was elevated in colon and serum [57]. The same study proved the benefits of targeting elastase in colitis models, though the mechanisms of these actions remain unclear [57]. In another study held by Amanzada et al, CD68(+) (a group of antigens on macrophages) elastase cells were identified as macrophagesmonocytes in human peripheral blood and intestinal tissue of IBD patients [4]. In the study by Locht et al in 30 patients with UC, no one was positive for antibodies against elastase [26]. Similar results were obtained in 30 patients with RA, of whom no one was detected with antibodies against elastase. Only one of $50(2 \%)$ patients with ReA was detected with elastase antibodies [31]. Contrary to the previous results, in the study of Bahari et al, mentioned before, of 108 Iranian patients with UC, auto-antibodies against elastase were detected in $25.9 \%$ [24]. Antibodies against elastase are detected in up to $35 \%$ of patients with PSC, according to Hov [17]. Lindgren et al examined 100 patients with $\mathrm{PBC}$, of whom $15 \%$ were positive for elastase, and 76 PSC patients, of whom $18.4 \%$ were positive for elastase.

Finally, the pANCA staining of antibodies to elastase is an artifact of the alcohol fixation of neutrophils, which causes positively charged cytoplasmic granular proteins to redistribute around the negatively charged nucleus. When neutrophils are fixed with formalin, pANCA staining of elastase is abolished and converted to cANCA (Table 5) [20].

\section{Concluding remarks}

The results of different studies reveal a clear association of these proteins with inflammatory actions, either pro- or antiinflammatory. Considering the example of lactoferrin, these actions can simultaneously exist in the same molecule [20]. Moreover, high levels of circulating lactoferrin imply active inflammatory disease $[34,36]$ and that makes it a promising marker reflecting the intensity of the inflammatory process in the intestine [3]. Cathepsin $\mathrm{G}$ could be useful for evaluating the activity of UC, as it exhibits higher titers in active than inactive phase of UC [55]. Because of their roles in host defense and disease activity, elastase and cathepsin $\mathrm{G}$ could be of interest as potential therapeutic targets [25]. BPI was also related 
to disease activity in UC patients, while in $\mathrm{CD}$ patients the relation referred to colonic involvement [30]. According to endoscopic and histopathological criteria, UC is more often associated with ANCA and ANCA proteins than CD, though an association with colonic CD also exists [17].

Very often, the diverse results arising from various research groups are also due to the differences among the patient populations examined and differences among the methods [20]. However, as long as the target antigen for each pathologic condition is still unidentified, IIF remains the only widely available technique for the detection of these antibodies [20]. Several studies imply that the production of these antibodies is probably a result of the immune tolerance loss, rather than an increased bowel permeability and the different serologic markers are not specifically related to disease activity $[11,20]$. Sensitive and specific solid-phase assays need to be developed, so that we can obtain more accurate results. Further characterization of such targets would probably yield important insight into the pathogenesis of IBD. The investigation of larger populations may also further define the role of autoantibodies as diagnostic tools.

\section{References}

1. Tesija Kuna A. Serological markers of inflammatory bowel disease. Biochem Medica 2013;23:28-42.

2. Roozendaal C, Kallenberg CGM, others. Are anti-neutrophil cytoplasmic antibodies (ANCA) clinically useful in inflammatory bowel disease (IBD)? Clin Exp Immunol 1999;116:206-213.

3. Moniuszko A, Wiśniewska A, Rydzewska G. Biomarkers in management of inflammatory bowel disease. Gastroenterol Rev 2013;5:275-283.

4. Amanzada A, Malik IA, Blaschke M, et al. Identification of CD68+ neutrophil granulocytes in in vitro model of acute inflammation and inflammatory bowel disease. Int J Clin Exp Pathol 2013;6:561.

5. Fournier BM, Parkos CA. The role of neutrophils during intestinal inflammation. Mucosal Immunol 2012;5:354-366.

6. Gionchetti P, Campieri M, Guarnieri C, et al. Respiratory burst of circulating polymorphonuclear leukocytes and plasma elastase levels in patients with inflammatory bowel disease in remission. Dig Dis Sci 1994;39:550-554.

7. Han N-Y, Kim EH, Choi J, et al. Quantitative proteomic approaches in biomarker discovery of inflammatory bowel disease: Biomarkers for IBD and colitic cancer. J Dig Dis 2012;13:497-503.

8. Terjung B, Spengler U, Sauerbruch T, Worman HJ. "Atypical p-ANCA" in IBD and hepatobiliary disorders react with a 50-kilodalton nuclear envelope protein of neutrophils and myeloid cell lines. Gastroenterology 2000;119:310-322.

9. Iskandar HN, Ciorba MA. Biomarkers in inflammatory bowel disease: current practices and recent advances. Transl Res 2012;159:313-325.

10. Selvaggi SM. Tumors of the ovary, maldeveloped gonads, fallopian tube, and broad ligament. Arch Pathol Lab Med 2000;124:474-474.

11. Papp M, Norman GL, Altorjay I, Lakatos PL. Utility of serological markers in inflammatory bowel diseases: gadget or magic? World J Gastroenterol 2007;13:2028-2036.

12. Mendoza JL, Abreu MT. Biological markers in inflammatory bowel disease: Practical consideration for clinicians. Inflamm Bowel Dis 2009;33(Suppl 3):S158-S173.

13. Vermeulen N, Vermeire S, Rutgeerts P, Bossuyt X. Serological markers in inflammatory bowel disease. Immuno-Anal Biol Spéc 2008;23:358-367.

14. Saxon A, Shanahan F, Landers C, et al. A distinct subset of antineutrophil cytoplasmic antibodies is associated with inflammatory bowel disease. J Allergy Clin Immunol 1990;86:202210.

15. Rump JA, Schölmerich J, Gross V, et al. A new type of perinuclear anti-neutrophil cytoplasmic antibody (p-ANCA) in active ulcerative colitis but not in Crohn's disease. Immunobiology 1990;181:406-413.

16. Reumaux D, Sendid B, Poulain D, et al. Serological markers in inflammatory bowel diseases. Best Pract Res Clin Gastroenterol 2003; 17:19-35.

17. Hov J-R. Autoantibodies in primary sclerosing cholangitis. World J Gastroenterol 2008; 14:3781.

18. Deniziaut G, Ballot E, Johanet C. Antineutrophil cytoplasmic auto-antibodies (ANCA) in autoimmune hepatitis and primary sclerosing cholangitis. Clin Res Hepatol Gastroenterol 2013;37:105-107.

19. Papp M, Altorjay I, Lakos G, et al. Evaluation of the combined application of ethanol-fixed and formaldehyde-fixed neutrophil substrates for identifying atypical perinuclear antineutrophil cytoplasmic antibodies in inflammatory bowel disease. Clin Vaccine Immunol 2009;16:464-470.

20. Bossuyt X. Serologic markers in inflammatory bowel disease. Clin Chem 2005;52:171-181.

21. Mosli M, Al Beshir M, Al-Judaibi B, et al. Advances in the diagnosis and management of inflammatory bowel disease: Challenges and uncertainties. Saudi J Gastroenterol 2014;20:81.

22. Lewis JD. The utility of biomarkers in the diagnosis and therapy of inflammatory bowel disease. Gastroenterology 2011;140:18171826; e2.

23. Fengming Y, Jianbing W. Biomarkers of inflammatory bowel disease. Dis Markers 2014;2014:1-11.

24. Bahari A, Aarabi M, Hedayati $M$, et al. Target antigens for perinuclear antineutrophil cytoplasmic antibodies in iranian patients with ulcerative colitis. Middle East J Dig Dis 2014;6:203.

25. Korkmaz B, Horwitz MS, Jenne DE, Gauthier F. Neutrophil elastase, proteinase 3, and cathepsin $G$ as therapeutic targets in human diseases. Pharmacol Rev 2010;62:726-759.

26. Ellrichmann M, Wietzke-Braun P, Wintermeyer L, et al. Endoscopic ultrasound of the sigmoid colon for the differentiation of Crohn's disease from unspecific, infectious colitis and healthy controls-a prospective, blinded, comparative study. Gastroenterology 2011;140:S-692.

27. Savige JA, Paspaliaris B, Silvestrini R, et al. A review of immunofluorescent patterns associated with antineutrophil cytoplasmic antibodies (ANCA) and their differentiation from other antibodies. J Clin Pathol 1998;51:568-575.

28. Stoffel MP, Csernok E, Herzberg C, et al. Anti-neutrophil cytoplasmic antibodies (ANCA) directed against bactericidal/ permeability increasing protein (BPI): a new seromarker for inflammatory bowel disease and associated disorders. Clin Exp Immunol 1996;104:54-59.

29. Schultz H, Csernok E, Schuster A, et al. Anti-neutrophil cytoplasmic antibodies directed against the bactericidal/ permeability-increasing protein (BPI) in pediatric cystic fibrosis patients do not recognize $\mathrm{N}$-terminal regions important for the anti-microbial and lipopolysaccharide-binding activity of BPI. Pediatr Allergy Immunol 2000;11:64-70.

30. Walmsley RS, Zhao MH, Hamilton MI, et al. Antineutrophil cytoplasm autoantibodies against bactericidal/permeabilityincreasing protein in inflammatory bowel disease. Gut 1997;40:105-109.

31. Locht H, Skogh T, Wiik A. Characterisation of autoantibodies to neutrophil granule constituents among patients with reactive 
arthritis, rheumatoid arthritis, and ulcerative colitis. Ann Rheum Dis 2000;59:898-903.

32. Esquivel-Valerio JA, Flores-Suárez LF, Rodríguez-Amado J, et al. Antineutrophil cytoplasm autoantibodies in patients with tuberculosis are directed against bactericidal/permeability increasing protein and are detected after treatment initiation. Clin Exp Rheumatol 2010;28:S35.

33. Mulder AH, Broekroelofs J, Horst G, et al. Anti-neutrophil cytoplasmic antibodies (ANCA) in inflammatory bowel disease: characterization and clinical correlates. Clin Exp Immunol 1994;95:490-497.

34. Peen E, Almer S, Bodemar G, et al. Anti-lactoferrin antibodies and other types of ANCA in ulcerative colitis, primary sclerosing cholangitis, and Crohn's disease. Gut 1993;34:56-62.

35. Levay PF, Viljoen M. Lactoferrin: a general review. Haematologica 1995;80:252-267.

36. Legrand D, Elass E, Carpentier M, Mazurier J. Lactoferrin: Lactoferrin: a modulator of immune and inflammatory responses. Cell Mol Life Sci 2005;62:2549-2559.

37. Locht H, Skogh T, Kihlström E. Anti-lactoferrin antibodies and other types of anti-neutrophil cytoplasmic antibodies (ANCA) in reactive arthritis and ankylosing spondylitis. Clin Exp Immunol 1999;117:568.

38. Sobajima J, Ozaki S, Okazaki T, et al. Anti-neutrophil cytoplasmic antibodies (ANCA) in ulcerative colitis: anti-cathepsin G and a novel antibody correlate with a refractory type. Clin Exp Immunol 1996;105:120-124.

39. Roozendaal C, Horst G, Pogány K, et al. Prevalence and clinical significance of anti-lactoferrin autoantibodies in inflammatory bowel diseases and primary sclerosing cholangitis. In Spik G, Legrand D, Mazurier J, et al. (eds): Adv Lact Res 1998;443:313-319.

40. Sugi K, Saitoh O, Matsuse R, et al. Antineutrophil cytoplasmic antibodies in Japanese patients with inflammatory bowel disease: prevalence and recognition of putative antigens. Am J Gastroenterol 1999;94:1304-1312.

41. Roozendaal C, de Jong MA, van den Berg AP, et al. Clinical significance of anti-neutrophil cytoplasmic antibodies (ANCA) in autoimmune liver diseases. J Hepatol 2000;32:734-741.

42. Teegen B, Niemann S, Probst C, et al. DNA-bound lactoferrin is the major target for antineutrophil perinuclear cytoplasmic antibodies in ulcerative colitis. Ann N Y Acad Sci 2009;1173:161-165.

43. Zhou X, Xu W, Tang X, et al. Fecal lactoferrin in discriminating inflammatory bowel disease from Irritable bowel syndrome: a diagnostic meta-analysis. BMC Gastroenterol 2014;14:121.

44. Kane SV, Sandborn WJ, Rufo PA, et al. Fecal lactoferrin is a sensitive and specific marker in identifying intestinal inflammation. Am J Gastroenterol 2003;98:1309-1314.

45. Buderus S, Boone JH, Lentze MJ. Fecal lactoferrin: reliable biomarker for intestinal inflammation in pediatric IBD. Gastroenterol Res Pract 2015 (Epub ahead of print).

46. D'Inca' R, Caccaro R. Measuring disease activity in Crohn's disease: what is currently available to the clinician. Clin Exp Gastroenterol 2014;20:151-161.

47. Gisbert JP, McNicholl AG. Questions and answers on the role of faecal calprotectin as a biological marker in inflammatory bowel disease. Dig Liver Dis 2009;41:56-66.

48. Dabek M, Ferrier L, Roka R, et al. Luminal cathepsin G and protease-activated receptor 4. Am J Pathol 2009;175:207-214.

49. Burster T, Macmillan H, Hou T, et al. Cathepsin G: roles in antigen presentation and beyond. Mol Immunol 2010;47:658-665.

50. Meyer-Hoffert U. Neutrophil-derived serine proteases modulate innate immune responses. Front Biosci Landmark Ed 2009;14:3409-3418.

51. Menzel K, Hausmann M, Obermeier F, et al. Cathepsins B, L and $\mathrm{D}$ in inflammatory bowel disease macrophages and potential therapeutic effects of cathepsin inhibition in vivo. Clin Exp Immunol 2006;146:169-180.

52. Miyata J, Tani K, Sato K, et al. Cathepsin G: the significance in rheumatoid arthritis as a monocyte chemoattractant. Rheumatol Int 2007;27:375-382.

53. Mayet WJ, Hermann E, Finsterwalder J, et al. Antibodies to Cathepsin G in Crohn's disease. Eur J Clin Invest 1992;22:427-433.

54. Sobajima J, Ozaki S, Osakada F, et al. Novel autoantigens of perinuclear anti-neutrophil cytoplasmic antibodies (P-ANCA) in ulcerative colitis: non-histone chromosomal proteins, HMG1 and HMG2. Clin Exp Immunol 1997;107:135-140.

55. Kuwana T, Sato Y, Saka M, et al. Anti-cathepsin G antibodies in the sera of patients with ulcerative colitis. J Gastroenterol 2000;35:682689.

56. Campbell EJ. Human leukocyte elastase, cathepsin G, and lactoferrin: family of neutrophil granule glycoproteins that bind to an alveolar macrophage receptor. Proc Natl Acad Sci 1982;79:6941-6945.

57. Shioya Y, Katakura K, Ohira H. Neutrophil elastase inhibitor suppresses IL-17 based inflammation of murine experimental colitis. Fukushima J Med Sci 2014;60:14-21.

58. Nässberger L, Ljungh A, Schumacher G, Kollberg B. $\beta$-glucuronidase antibodies in ulcerative colitis. Orig Publ 1992;340:734-735.

59. Broekroelofs J, Mulder AHL, Nelis GF, et al. Anti-neutrophil cytoplasmic antibodies (ANCA) in sera from patients with inflammatory bowel disease (IBD). Dig Dis Sci 1994;39:545-549.

60. Kossa K, Coulthart A, Ives C, et al. Antigen specificity of circulating anti-neutrophil cytoplasmic antibodies in inflammatory bowel disease. Eur J Gastroenterol Hepatol 1995;7:783-789.

61. Yang P, Bohr J, Tysk C, et al. Antineutrophil cytoplasmic antibodies in inflammatory bowel disease and collagenous colitis: no association with lactoferrin, $\beta$-glucuronidase, myeloperoxidase, or proteinase 3. Inflamm Bowel Dis 1996;2:173-177.

62. Sobajima J, Ozaki S, Uesugi H, et al. Prevalence and characterization of perinuclear anti-neutrophil cytoplasmic antibodies (P-ANCA) directed against HMG1 and HMG2 in ulcerative colitis (UC). Clin Exp Immunol 1998;111:402-407.

63. Roozendaal C, Zhao MH, Horst G, et al. Catalase and $\alpha$-enolase: two novel granulocyte autoantigens in inflammatory bowel disease (IBD). Clin Exp Immunol 1998;112:10-16.

64. Lindgren S, Nilsson S, Nässberger L, et al. Anti-neutrophil cytoplasmic antibodies in patients with chronic liver diseases: prevalence, antigen specificity and predictive value for diagnosis of autoimmune liver disease. Swedish Internal Medicine Liver Club (SILK). J Gastroenterol Hepatol 2000;15:437-442. 